\title{
Creatine Phosphokinase Increased
}

National Cancer Institute

\section{Source}

National Cancer Institute. Creatine Phosphokinase Increased. NCI Thesaurus. Code C78247.

A laboratory test result which indicates increased levels of creatine phosphokinase in a biological specimen. 